\title{
Research on Damage Identification of Grid Structure Based on Genetic Algorithm
}

\author{
Yu Wang ${ }^{1 *}$, Menghong Wang ${ }^{1}$ and Huan $\mathrm{Lu}^{2}$ \\ ${ }^{1}$ School of Civil and Transportation Engineering, Beijing University of Civil Engineering and Architecture, Beijing, 100044, China \\ ${ }^{2}$ Hebei Institute Of Architectural Design\&Research CO., LTD, Shijiazhuang, Hebei, 050000, China
}

\begin{abstract}
This paper proposes a genetic algorithm based damage identification method for grid structures. The genetic algorithm is used to process the modal information of the structure, and the damage identification of the truss structure is carried out. The stiffness reduction factor of the structural member is used as the optimization variable. The objective function is constructed according to the frequency and vibration mode, and the fitness function is established. The binary coding method is used to improve the crossover and mutation operators. In this paper, a grid structure model is used for numerical simulation analysis and verified by experiments. In the experimental stage, the grid structure is excited by hammering method, and the response data of each node and the modal information of the structure are obtained. Numerical simulation and experimental analysis show that the damage identification method based on genetic algorithm can effectively judge the location and extent of damage.
\end{abstract}

\section{Introduction}

The grid structure tends to withstand some large loads during service, and may cause damage such as loose bolts, bending or even fracture of the rods. These damages are often difficult to observe by visual inspection. The local damage accumulation of the structure and the reduction of the resistance are inevitable in the service process. If the damage accumulation of the key components is not detected in time, the damage will rapidly expand, resulting in damage to the entire structure. In order to ensure the safety of the grid structure during the service period, it is necessary to detect and identify the damage of the structure.

The structure will produce corresponding response under the action of excitation. We can select the corresponding key indicators through the response information of the in-service structure to judge whether the structure has damage [1-2].

Genetic algorithm is a randomized global search algorithm based on biological natural selection and natural genetic mechanism. It transforms the damage identification problem of the structure into a numerical optimization problem, mainly by optimizing the objective function and finally obtaining the global optimal value [3]. This method has many advantages: 1) it has a strong global search capability. Genetic algorithm searches from the initial population with feasible solution, which has a larger search range and is more conducive to global search and optimization. 2) It has a wide range of applications. The genetic algorithm is not limited by the complexity of the problem in calculation, and does not require the objective function to satisfy the conditions of continuous and differentiability [4].

\section{Application of genetic algorithm in structural damage identification}

The analysis process of genetic algorithm includes the coding scheme of parameters, fitness function, selection, hybridization, mutation and other basic operations, termination criteria and constraints [5].

\subsection{Encoding and decoding methods}

The stiffness reduction factor is encoded by a binary coding method and converted into a binary string. The length of the code string depends on the accuracy required by the problem. The rod unit stiffness reduction factor $\alpha$ has a value range of $[0,1]$, with 0 representing the intact state and 1 representing the complete damage state. If the accuracy requirement is accurate to 4 digits after the decimal point, then each variable should be divided into at least $(1-0) \times 10^{4}$ parts. The number of binary strings for a variable can be calculated using the following formula:

$$
2^{m_{j}-1}<(1-0) \times 10^{4} \leq 2^{m_{j}}-1
$$

This solution: $m_{j}=14$, that is, a chromosome string is 14 bits.

The variable is binary coded and needs to be decoded to output a decimal value when outputting the variable.

*Corresponding author's e-mail: wangyu5320@163.com 
Returning a decimal value from a binary string can be implemented using the following formula:

$$
\alpha=0+\operatorname{decimal}\left(\text { substring }_{j}\right) \times \frac{1-0}{2^{m_{j}}-1}
$$

Where decimal(substring ${ }_{j}$ ) represents the decimal value of the variable $\alpha$ obtained by binary conversion.

\subsection{Objective function and fitness function}

In this paper, we choose the weighted sum of the difference between the analytical value and the measured value of frequency and mode to construct the objective function [6].

$$
\begin{gathered}
f_{\infty}(\alpha)=\sum_{i=1}^{n}\left(\frac{\omega_{u i}-\omega_{d i}}{\omega_{u i}}\right)^{2} \\
f_{\phi}(\alpha)=1-\sum_{i=1}^{j} \frac{\left(\left\{\phi_{u i}\right\}^{T}\left\{\phi_{d i}\right\}\right)^{2}}{\left(\left\{\phi_{u i}\right\}^{T}\left\{\phi_{d i}\right\}\right)\left(\left\{\phi_{u i}\right\}^{T}\left\{\phi_{d i}\right\}\right)}
\end{gathered}
$$

Where $\omega_{u i}$ and $\omega_{d i}$ are the analysis and measured values of the $\mathrm{i}$-th natural frequency of the structure respectively; $\alpha$ is the row vector representing the element damage factor; $\left\{\phi_{u i}\right\}$ and $\phi_{d i}$ respectively represent the analytical and measured values of the i-th mode of the structure. The objective function can be expressed as:

$$
f(x)=C_{\omega} f_{\omega}(\alpha)+C_{\phi} f_{\phi}(\alpha)
$$

Where $C_{\omega}$ and $C_{\phi}$ are weighting factors that measure the degree of accuracy between the natural frequency and the mode; $C_{\omega}=1, C_{\phi}=0.1$.

Then establish the fitness function Fit $(x)$ by the boundary construction method:

$$
F i t(x)=\frac{1}{1+c+f(x)}, c \geq 0, c+f(x) \geq 0
$$

Where $c$ is Conservative estimate of the bounds of the objective function.

\subsection{Select}

In this paper, the roulette selection method is adopted. The probability that an individual is selected is equal to the ratio of its fitness value to the total fitness value of the population. Assuming that there are $\mathrm{n}$ individuals in a certain group, the probability that a certain body enters the next generation can be expressed as:

$$
p_{i}=f / \sum_{i=1}^{n} f_{i}
$$

Where $p_{i}$ is the probability that the individual will enter the next generation of the population; $f$ is the fitness value of the individual; $f_{i}$ is the fitness value of a certain body of the population.

\subsection{Cross and variation}

Individuals with a fitness value higher than the average fitness value of the group should be given a lower probability of crossover and mutation to maintain their superiority; otherwise, the probability of crossover and mutation should be increased, so that the individual has a greater probability of evolution [7]. The corresponding parameter improvement formula can be expressed as:

$$
\begin{aligned}
& p_{c}=\left\{\begin{array}{c}
\frac{k_{1}\left(f_{\max }-f\right)}{f_{\max }-f_{\text {avg }}}, f \geq f_{\text {avg }} \\
k_{2}, f<f_{\text {avg }}
\end{array}\right. \\
& p_{m}=\left\{\begin{array}{c}
\frac{k_{3}\left(f_{\max }-f\right)}{f_{\max }-f_{\text {avg }}}, f \geq f_{\text {avg }} \\
k_{4}, f<f_{\text {avg }}
\end{array}\right.
\end{aligned}
$$

Where $p_{c}$ is the crossover probability; $p_{m}$ is the probability of variation; $f_{\max }$ is the maximum target value in the contemporary group; $f_{\text {avg }}$ is the average target value of contemporary groups; $f$ is the larger target value of the two intersecting individuals; $f^{\prime}$ is the target value of the mutant; $k_{1}, k_{2}, k_{3}, k_{4}$ is a constant.

\section{Damage identification numerical simulation}

\subsection{Numerical analysis model}

The model used in the numerical simulation of this paper is a shell-and-grid structure. The grid structure model has 60 nodes and 174 rods with a size of $3 \mathrm{~m} \times 5 \mathrm{~m}$. The structural members are all produced with Q235 steel pipe, the modulus of elasticity is $206 \mathrm{GPa}$, and the crosssection dimensions of the bars are $\Phi 42 \mathrm{~mm} \times 2.5 \mathrm{~mm}$.The truss structure is fixedly connected to the foundation at four supports, and the connection between the nodes is hinged [8]. The grid structure model and the part number are shown in Figure 1.

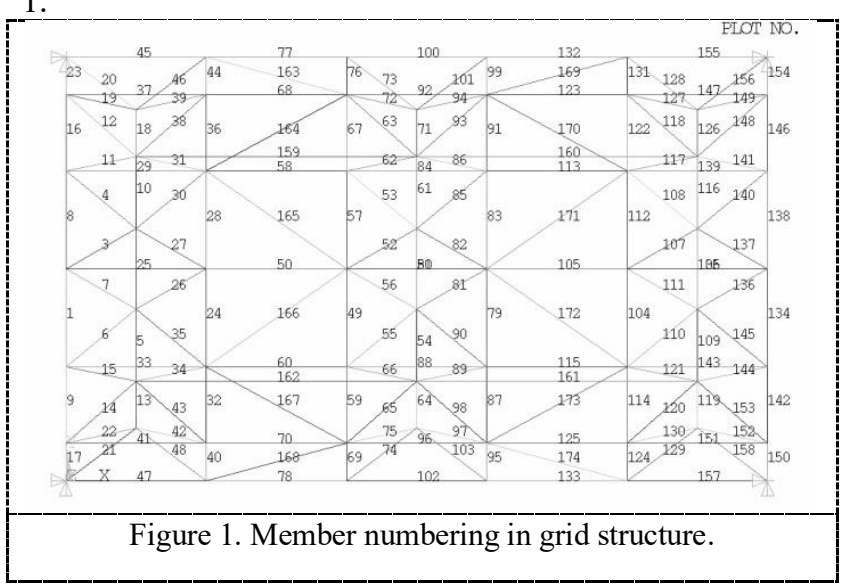




\subsection{Working condition setting}

In order to simulate the different positions of the truss structure and different degrees of damage, three different working conditions are set.

Working condition 1: non-destructive structure;

Working condition 2: the structure of the lower chord - the 106th member (the two ends of the node is No. 43 and No. 44) is completely damaged, that is, the rod is broken and the rigidity is 0 ;

Working condition 3: the structure of the chord - the 28th member (the two ends of the node is No. 14 and No. 15 ) is $50 \%$ damaged.

\subsection{Identification results and analysis}

For the simulation analysis of damage identification of working condition 2, firstly, the parameter values in adaptive crossover probability and mutation probability need to be set. $k_{1}=0.7, k_{2}=0.8, k_{3}=0.6, k_{4}=0.35$, The termination evolution algebra of the genetic algorithm is set to 170 . Due to the space limitation, the analysis results of the listed partial units are shown in Table 1.

Table 1. Damage identification result under condition II.

\begin{tabular}{cccccc}
\hline Unit & $\alpha$ & Unit & $\alpha$ & Unit & $\alpha$ \\
\hline 101 & 0.0335 & 111 & 0.0593 & 121 & 0.0107 \\
102 & 0.0512 & 112 & 0.0324 & 122 & 0.0216 \\
103 & 0.0209 & 113 & 0.0424 & 123 & 0.0034 \\
104 & 0.0268 & 114 & 0.0600 & 124 & 0.0931 \\
105 & 0.0033 & 115 & 0.0173 & 125 & 0.0202 \\
106 & 0.9954 & 116 & 0.0249 & 126 & 0.0105 \\
107 & 0.0398 & 117 & 0.0279 & 127 & 0.0125 \\
108 & 0.1320 & 118 & 0.0458 & 128 & 0.0543 \\
109 & 0.0539 & 119 & 0.0491 & 129 & 0.0405 \\
110 & 0.0071 & 120 & 0.0060 & 130 & 0.0281 \\
\hline
\end{tabular}

As can be seen from the table, the 106 member recognizes a large damage, and the damage value of the member is $99.54 \%$, which is closer to complete damage. The other members of the structure have no major damage and the recognition effect is good.

Subsequently, the damage identification simulation analysis of the working condition 3 is carried out, and the parameters of the genetic algorithm also need to be set first. Since the damage of the rod is relatively small, the adaptive crossover probability and the mutation probability are increased, and $k_{1}=0.7, k_{2}=0.87, k_{3}=0.6, k_{4}=0.5 \quad$ are $\quad$ set respectively; the population size is set to 60 ; and the termination evolution algebra of the genetic algorithm is set to 180 . Due to space limitations, the analysis results of some of the listed units are shown in Table 2.

Table 2. Damage identification result under condition III.

\begin{tabular}{cccccc}
\hline Unit & $\alpha$ & Unit & $\alpha$ & Unit & $\alpha$ \\
\hline
\end{tabular}

\begin{tabular}{cccccc}
\hline 1 & 0.0119 & 11 & 0.0369 & 21 & 0.0241 \\
2 & 0.0117 & 12 & 0.0226 & 22 & 0.0421 \\
3 & 0.0196 & 13 & 0.0526 & 23 & 0.0226 \\
4 & 0.0528 & 14 & 0.0471 & 24 & 0.0441 \\
5 & 0.0283 & 15 & 0.0279 & 25 & 0.0272 \\
6 & 0.0242 & 16 & 0.0488 & 26 & 0.0326 \\
7 & 0.0108 & 17 & 0.0139 & 27 & 0.0124 \\
8 & 0.0581 & 18 & 0.0258 & 28 & 0.4932 \\
9 & 0.0244 & 19 & 0.0201 & 29 & 0.0043 \\
10 & 0.0119 & 20 & 0.0358 & 30 & 0.0109 \\
\hline
\end{tabular}

As can be seen from the table, the 28th member can observe a large damage. The above simulation analysis shows that the damage identification method based on genetic algorithm can effectively identify the location and extent of damage in the structure.

\section{Damage identification experiment analysis}

\subsection{Grid structure experimental model}

The experimental model is produced and assembled with reference to the numerical analysis model. The dimensions of the specific members and structures are shown in the numerical simulation analysis section. The experimental model of the grid structure is shown in Figure 2 .

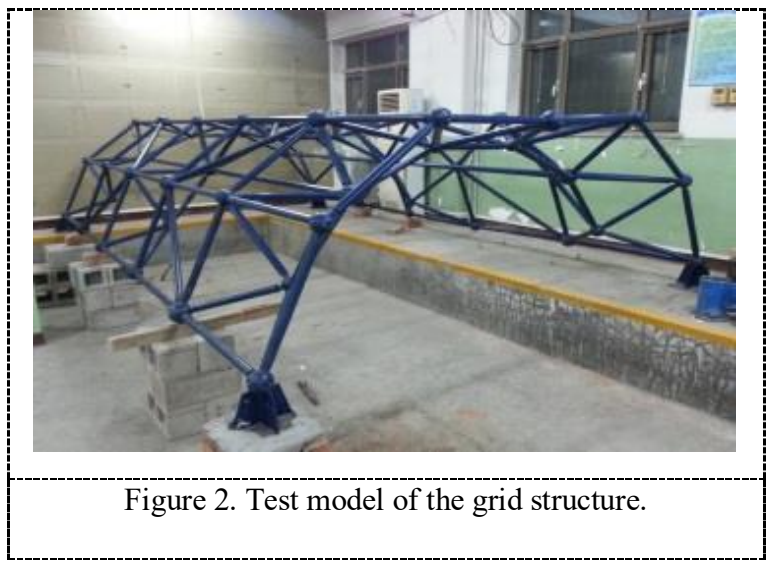

\subsection{Analysis of experimental results}

The non-destructive working condition and the complete damage of the 34th web member are set, that is, the stiffness is reduced by $100 \%$, and the two ends are respectively No. 6 and No. 14.The corresponding parameter values of the genetic algorithm are set as follows: crossover and mutation probability: $k_{1}=0.7, k_{2}=0.75, k_{3}=0.6, k_{4}=0.4 \quad ; \quad$ the population size is set to 70 , which can ensure the search efficiency while ensuring the optimal result; set the termination evolution of the genetic algorithm, The algebra is 200.The analysis results of some of the bars are listed here, as shown in Table 3. It can be found from 
the table that the damage identification value of the No. $34 \operatorname{rod}$ is $99.31 \%$.

Table 3. Damage identification result.

\begin{tabular}{cccccc}
\hline Unit & $\alpha$ & Unit & $\alpha$ & Unit & $\alpha$ \\
\hline 21 & 0.0915 & 31 & 0.0494 & 156 & 0.0943 \\
22 & 0.0639 & 32 & 0.0948 & 157 & 0.0911 \\
23 & 0.0941 & 33 & 0.0278 & 158 & 0.0347 \\
24 & 0.0854 & 34 & 0.9931 & 159 & 0.0578 \\
25 & 0.0652 & 35 & 0.0734 & 160 & 0.2183 \\
26 & 0.1244 & 36 & 0.1770 & 161 & 0.0226 \\
27 & 0.1358 & 37 & 0.0529 & 162 & 0.0422 \\
28 & 0.0810 & 38 & 0.0644 & 163 & 0.0756 \\
29 & 0.0307 & 39 & 0.0478 & 164 & 0.0503 \\
30 & 0.0585 & 40 & 0.0899 & 165 & 0.0871 \\
\hline
\end{tabular}

It can be seen from Table 3 that the No. 34 rods identify more obvious damages, but other rods also have misidentification. Among them, the No. 160 nondestructive rods have the largest identification error, reaching $21.83 \%$.

\section{Conclusion}

In this paper, the damage identification method of grid structure based on genetic algorithm is studied. The effectiveness of the method is verified by numerical simulation and experimental analysis. The main conclusions are as follows:

(1) The damages of different positions and degrees of the truss structure were identified, and the two conditions of the complete damage of the 106th chord member and the partial damage of the 28th chord member were respectively set. Numerical simulation analysis shows that the genetic algorithm can effectively identify the location and extent of damage for structural damage conditions.

(2) Through the setting of the damage condition of the single member of the grid structure, the damage analysis of the grid structure was analyzed experimentally. The experimental results show that the damage identification method based on genetic algorithm can effectively identify the condition of complete damage of the single member of the grid structure.

(3) Through experimental analysis, it can be found that there will be some misidentification in the results. The first reason is the influence of environmental noise. The second is that there is a certain difference between the experimental modal information and the numerical simulation value, and the error appears.

\section{References}

1. Ren WX, Guido De Roeck. (2002) Structural damage identification using modal data I: Simulation verification. Journal of Structural Engineering. Vol.128 (1), pp.87-95.
2. Sun XD, Ou JP. (2009)Assessment of vibration based damage indexes in structural health monitoring $[\mathrm{J}]$. Journal of Vibration and Shock. 28(01): 9-13+191.

3. Srinivas M, Patnaik LM. (1994) Adaptive probabilities of crossover and mutation in genetic algorithms. IEEE Transactions on Systems Man and Cybernetics.

4. Yi WJ, LIU X. (2001) Damage diagnosis of structures by genetic algorithms $[\mathrm{J}]$. Engineering Mechanics. (02):64-71.

5. Wang XP, Cao LM. (2002) Genetic Algorithm Theory, Application and Software Implementation[M]. Xi'an: Xi'an Jiaotong University Press. Xi'an.

6. Yuan Y, Lin G, Liu CG, Zhou AH. (2005) Study on an Application of Genetic Algorithm to Damage Identification[J]. Journal of Disaster Prevention and Mitigation Engineering. (04):369-374.

7. Huang MS, WU L, ZHU HP. (2012) Structural damage identification based on improved damage identification factor and genetic algorithm under noise $[\mathrm{J}]$. Journal of Vibration and Shock. 31(21):168-174.

8. Wang XM. (2007) ANSYS engineering structure numerical analysis[M]. People's Communications Publishing House. Beijing. 